\title{
Tumor Infiltrating Lymphocytes in Ovarian Cancer
}

\author{
Maria Luisa Gasparri ${ }^{1 *}$, Rukset Attar ${ }^{2}$, Innocenza Palaia ${ }^{1}$, Giorgia Perniola ${ }^{1}$, \\ Claudia Marchetti ${ }^{1}$, Violante Di Donato ${ }^{1}$, Ammad Ahmad Farooqi ${ }^{3}$, Andrea \\ Papadia $^{4}$, Pierluigi Benedetti Panici ${ }^{1}$
}

\begin{abstract}
Several improvements in ovarian cancer treatment have been achieved in recent years, both in surgery and in combination chemotherapy with targeting. However, ovarian tumors remain the women's cancers with highest mortality rates. In this scenario, a pivotal role has been endorsed to the immunological environment and to the immunological mechanisms involved in ovarian cancer behavior. Recent evidence suggests a loss of the critical balance between immune-activating and immune-suppressing mechanisms when oncogenesis and cancer progression occur. Ovarian cancer generates a mechanism to escape the immune system by producing a highly suppressive environment. Immune-activated tumor infiltrating lymphocytes (TILs) in ovarian tumor tissue testify that the immune system is the trigger in this neoplasm. The TIL mileau has been demonstrated to be associated with better prognosis, more chemosensitivity, and more cases of optimal residual tumor achieved during primary cytoreduction. Nowadays, scientists are focusing attention on new immunologically effective tumor biomarkers in order to optimize selection of patients for recruitment in clinical trials and to identify relationships of these biomarkers with responses to immunotherapeutics. Assessing this point of view, TILs might be considered as a potent predictive immunotherapy biomarker.
\end{abstract}

Keywords: Tumor infiltrating lymphocytes - cancer - apoptosis - target therapy

Asian Pac J Cancer Prev, 16 (9), 3635-3638

\section{Introduction}

Ovarian cancer is the gynecological malignancies with the highest mortality, ranging a median overall survival of about 30\% (Heintz et al., 2006). Despite considerable progress in sugical field (Angioli et al., 2013; Papadia et al., 2013) and conventional chemotherapy associated with target therapies (Marchetti et al., 2012; Ferrero et al., 2013), not sufficient resolutive strategies have been achieved. Thus, this neoplasm remains a challenge disease for gynecologic oncologist, due to the high proportion of patients suffering from disease progression during primary treatment and recurrence disease. These failures can be ascribed to the incapability of standard treatment strategies to completely eradicate local and/or metastatic tumors. Based on recent findings, a speculative correlation with this aggressive behavior can be ascribed to the immune system not only during cancer progression, but also how mechanistically neoplastic lesions respond to wide ranging therapeutic interventions (Curiel et al., 2004). In this scenario, accurate investigations on tumor cell network and tumor microenvironment are mandatory.

The recruitment of immunosuppressive factors has been implemented by tumor tissue in order to evading immune recognition, through production of cytokines and $\mathrm{T}$ regulatory cells, which lead to the basis of the immunosuppressive milieu that allows cancer immune evasion and reduced numbers and impaired functions of dendritic cells (Mougiakakos et al 2010; Gasparri et al., 2013). In ovarian cancer, the amount of this cell subset highly increased in peripheral blood and in tumor (Napoletano et al., 2010) and a low ratio of tumor infiltrating CD8/Treg cells is correlated to a worst prognosis (Sato et al., 2005). Conversely, other subset of T lymphocyte (e.g. T CD8 effector) plays a dominant role in direct lysis of targeted host cells. The "cancer immunoeditingtheory"establishes that tumors arise from a failure of the immune system, occurring after a disequilibrium between tumor growth and immune surveillance (Schreiber et al., 2011). This interaction between immune system and tumor progression represents the key point in different cancer behavior (Ygit et al., 2010).

Lymphocytes infiltrating tumor (TILs) analysis demonstrated to have a strong prognostic and predictive role in several malignancies, including gynecological neoplasms, with particularly ovarian cancer (Table 1A, Table 1B). 
Table 1A. Tumor associated lymphocyte in Ovarian Cancer

\begin{tabular}{lclc}
\hline Authors & Type of cancer & Infiltrate population & Prognostic Association \\
\hline Stato et al, Proc Nact Acad Sci USA 2005 & Ovarian & Intratumoral CD8 & Positive \\
& & None & None \\
& & Intratumoral CD3 & Positive \\
Clarke et al,Mod Patol 2009 & Ovarian & Intratumoral CD8 & None \\
& & Intratumoral CD3 & Positive \\
Zhang et al, N Eng J Med 2003 & Ovarian & Intratumoral CD3 & Negative \\
Curiel et al, Nat Med 2004 & Ovarian & Tumor Infiltrating Treg & Positive \\
Tomsova et al, Gynecol Oncol 2007 & Ovarian & Intratumoral CD3 & None \\
& & Peritumoral CD3 & Positive \\
Leffers et al, Cancer Immunol Immunother 2009 & Ovarian & Intratumoral CD8 & Positive \\
& & Intratumoral Treg & None \\
Shah et al, Gynecol Oncol 2008 & Ovarian & Intratumoral lymphocyte & Positive \\
Hamanishi et al, Proct Natl Acad Sci USA 2007 & Ovarian & Intratumoral CD8 & Positive \\
Stumpf et al, Br J Cancer 2009 & Ovarian & Intratumoral CD8 & None \\
& & Peritumoral CD8 & \\
\hline
\end{tabular}

Table 1B. Tumor Associated Lymphocyte in Other Gynecological Cancer

\begin{tabular}{lccc}
\hline Authors & Type of cancer & Infiltrate population & Prognostic Association \\
\hline Aaltomaa et al, Eur J Cancer 1992 & Breast & Tumor Infiltrating lymphocyte & Positive \\
Rahka et al, Eur J Cancer 2009 & Breast & Tumor Infiltrating lymphocyte & Positive \\
Kondratiev et al, Clin Cancer Res 2004 & Endometrial & Intratumoral CD8 & Positive \\
De Jong et al, Gynecol Oncol 2009 & Endometrial & Intratumoral CD8 & Positive \\
\hline
\end{tabular}

\section{Interaction between Immune System and Ovarian Cancer Treatment}

Recent evidences suggest that conventional treatment can partially restore the equilibrium between tumor progression and immune surveillance, key factors of tumor escape. Primary cytoreduction has been associated with a significant depletion of circulating $\mathrm{T}$ lymphocyte with immunosuppressive activity (T-regulatory cells) and a quantitative and qualitative increase in CD8 T-cell (Napoletano et al., 2010). This finding supports the thesis that surgical debulking plays also a beneficial systemic effect by reverting immunosuppression and restoring immunological fitness. Accumulating evidences indicate that also successful antineoplastic agents exert therapeutic effects by immune responses (Zitvogel et al., 2011), because neoplastic cells can become immunogenic as consequence of chemotherapy (Kroemer et al., 2013). The trigger is the release of additional cancer antigens from dying neoplastic cells. Recently, it has been described paradigm system in ovarian cancer, in order to identify chemotherapy associated antigens (CAAs) evaluating the specific $\mathrm{T}$ cell subsets that recognized antigens associated with apoptotic cells expressing these antigens. These chemotherapeutics- induced memory T-cell responses against CAAswere associated with prolonged survival in response to chemotherapy (Barnaba et al., 2014). Based on these relevant results, additional assays of immune system behavior during chemotherapy could be performed by pathologist through a quantitative and qualitative analysis of the subclass of TILs.

\section{Tumor Infiltrating Lymphocyte (TILs)}

Increasingly it is being realized that CD4+ TIL significantly expressed CD25, however, CD8+ TIL negligibly expressed CD25. More importantly, CD25+FoxP3- T cells showed a strong positive correlation with survival of patients (deLeeuw et al., 2014). It has recently been convincingly revealed that SMARCE1 and TTF1 mRNA expression markedly correlated with the number of intra-tumoral CD8+ cells. Overexpression of SMARCE1 in ovarian SKOV3 cancer cells induced secretion of different chemokines including IL8, RANTES and MIP1b in the supernatant and promoted CD8+ lymphocytes chemotaxis (Giannakakis et al., 2014). Artificial antigen-presenting cells (aAPC) equipped with the co-stimulatory molecule $4-1 \mathrm{BBL}, \mathrm{Fc}-\gamma$ receptor, , and secreted either IL-2, IL-21, or IL-15 cytokine. Greatest overall expansion of TIL was noted by IL-2 secreting aAPC. CD3+ T cells were considerably higher and CD3CD56+ NK cells were lower in IL-2 aAPC-expanded TIL (Santegoets et al., 2013). There is an exciting piece of evidence suggesting that patient survival rate was better in patients having CD20(+) and CD8(+) TIL as compared to those expressing CD8(+) TIL alone (Nielsen et al, 2012). Interestingly, data from another study suggests that higher infiltration of CD8+ cells correlates with improved overall survival (Bachmayr-Heyda et al., 2013).

Assuming that lymphocytic tumor infiltrate reflect the tumor specific immune response, TILs represent a potential marker of the intensity of the immune response to cancer. T-lymphocyte microenvironment attends a positive prognosis in several neoplasm, including gynecological malignancies (Table 1A, 1B). At date, the College of American Pathologists recommends that a characterization of the tumor infiltrating and peritumoral or systemic immune response should be investigated as a essential aspect during clinical evaluation of melanoma (Clemente et al., 1996), colorectal carcinoma (Salama et al., 2010) 
and Merkel cell carcinoma (Paulson et al., 2010). In these tumors, a clinical significance to TILs classification has been translated in worse or better prognosis, on the basis of absence of these $\mathrm{T}$ cells within the tumor or only focal presence or not along entire base of vertical growth phase (worse prognosis). In hepatocellular carcinoma patients with metastasis less than 12 months, CD8+T cells and NK cells frequencies in TILs were lower than those without metastasis (Guo et al, 2012).It is relevant to mention that diffusely infiltrated lymphocytes into entire invasive component or entire base of vertical growth phase are correlated with better prognosis.

\section{TILs in Ovarian Cancer Tissue}

Tumor-infiltrating lymphocytes have been significantly represented into tumoral ovarian tissue, supporting an effective interaction between the host's immune system and cancer. In epithelial cancers, tumor infiltrating lymphocytes are strictly correlated with overall survival (Jochems et al., 2011).

More significant correlation has been reported when CD8+ TILs are located within the neoplastic epithelium, rather than in the tumor-associated stroma (Clarke et al., 2009). Some authors investigated the mechanisms promoting these particular recruitments and localizations (Webb et al., 2014). Recently, it has been investigated a specific marker (CD103) of antigen-specific T-cells demarcating TILs presenting in epithelial tissue that seem to mediate protective antitumor immunity, consisting in highly activated, cytolytic CD8 TILs (Webb et al., 2014). The intra-epithelial marker CD103 signed TILS that abundantly infiltrate ovarian cancer and strongly correlate with increased disease-specific survival.In a recent metanalysis (Wei-Ting Hwang et al., 2012), data arising from ten studies comprising 1,815 patients with ovarian cancer, were immunologically analyzed. A lack of intraepithelial TILs has been showed to be significantly associated with a worse survival (HR, 2.24, 95\% CI, 1.71-2.91). Particularly, this analysis also shows that the prognostic value of TILs may vary depending on surgical outcomes. Interesting, the effects associated with TILs were discordant when optimally debulked cases have been considerated compared to studies including suboptimally de-bulked patients. Furthermore, Zhang et al (Zhang et al., 2003) shows that TIL positive women were more likely to have been optimally cytoreduction, suggesting the speculative hypotesis that anti-tumor immune responses may have contained disease spread or restrained tumor biology facilitating surgical de-bulking. In any cases, if further statistical analysis confirm this difference, TILs could be useful to direct surgical decision-making or neoadjuvant chemotherapy. Regarding chemotherapy treatment, a positive correlation between CD3+ TILs and clinical response rate to first-line chemotherapy was reported in two studies. (Raspollini et al., 2005, Tomsova et al., 2008)

\section{Future Prospectives}

Recent evidence is emphasizing on most advanced proteomics technologies to identify diagnosis biomarkers for different cancers (Luo et al, 2014). Multi-tumor marker protein chips are currently being used for ovarian cancer diagnosis and selection of treatment options (Bian et al, 2014). New information regarding unresolved mechanism involved in the interactions between tumor cells and immune system are mandatory, such as investigate and monitor $\mathrm{T}$ cell phenotype and activity not only in particularly recruitment tissue districts (e.g. lymph nodes and ascites), but also into tumor issue, correlating with standard therapies response. Different T cell populations (effector memory, central memory, naïve cells and $\mathrm{T}$ regulatory) should be studied at diagnosis (arising from tissue biopsy, if available), after surgery (arising from tumor sample), and at every course of chemotherapy (circulating $\mathrm{T}$ cells) in order to define the importance of $\mathrm{T}$ cells subset at different stages of tumor progression, and to define if treatment can be effective in reducing $\mathrm{T}$ regulatory cells (and T CD4+ producing IL17) and if $\mathrm{T}$ regulatory depletion is essential to achieve an immune-reversion. Furthermore, specific markers such as CD103, could be used to select specific TIL subsets for further antigens discoveries, response monitoring, and therapeutic interventions. Finally, indicate parameters of immune fitness could be essential for monitoring the immunological status of patients affected by ovarian cancer and for performing the optimal time to vaccination during current standard therapy in order to increase immune activation. A characterization of the lymphocyte response, and particularly of tumor infiltrating lymphocyte, might prove to be useful for a deeper analysis and to get a step closer to personalized medicine.

\section{References}

Bachmayr-Heyda A, Aust S, Heinze G, et al (2013). Prognostic impact of tumor infiltrating CD8+ T cells in association with cell proliferation in ovarian cancer patients--a study of the OVCAD consortium. BMC Cancer, 13, 422.

Bian J, Li B, Kou XJ, et al (2014). Clinical applicability of multitumor marker protein chips for diagnosing ovarian cancer. Asian Pac J Cancer Prev, 15, 8409-11.

Heintz AP, Odicino F, Maisonneuve P, et al (2006). Carcinoma of the ovary. FIGO 26th Annual report on the results of treatment in gynecological cancer. Int J Gynaecol Obstet, 95, 161-92.

Clarke B, Tinker AV, Lee CH, et al (2009). Intraepithelial T cells and prognosis in ovarian carcinoma, novel associations with stage, tumor type, and BRCA1 loss. Mod Pathol, 22, 393-402.

Clemente CG, Mihm MC Jr, Bufalino R, et al (1996). Prognostic value of tumor infiltrating lymphocytes in the vertical growth phase of primary cutaneous melanoma. Cancer, 77, 1303-10.

deLeeuw RJ, Kroeger DR, Kost SE, et al (2014). CD25 identifies a subset of CD4+FoxP3-TIL that are exhausted yet prognostically favorable in human ovarian cancer. Cancer Immunol Res, 2014.

Gasparri ML, Bellati F, Napoletano C, et al (2013). Interaction between Treg cells and angiogenesis, a dark double track. Int J Cancer, 132, 2469.

Giannakakis A, Karapetsas A, Dangaj D, et al (2014). Overexpression of SMARCE1 is associated with CD8+ 
Maria Luisa Gasparri et al

T-cell infiltration in early stage ovarian cancer. Int J Biochem Cell Biol, 53, 389-98.

Guo CL, Yang HC, Yang XH, et al (2012). Associations between infiltrating lymphocyte subsets and hepatocellular carcinoma. Asian Pac J Cancer Prev, 13, 5909-13.

Hwang WT1, Adams SF, Tahirovic E, et al (2012). Prognostic significance of tumor-infiltrating T cells in ovarian cancer, a meta-analysis. Gynecol Oncol, 124, 192-8.

Jochems C1, Schlom J (2011). Tumor-infiltrating immune cells and prognosis, the potential link between conventional cancer therapy and immunity. Exp Biol Med, 236, 567-79.

Kroemer G1, Galluzzi L, Kepp O, et al (2013). Immunogenic cell death in cancer therapy. Annu Rev Immunol, 31, 51-72.

Leone Roberti Maggiore U, Bellati F, Ruscito I, Gasparri ML, et al (2013). Monoclonal antibodies therapies for ovarian cancer. Expert Opin Biol Ther, 13, 739-64.

Luo L, Dong LY, Yan QG. (2014) Research Progress in Applying Proteomics Technology to Explore Early Diagnosis Biomarkers of Breast Cancer, Lung Cancer and Ovarian Cancer. Asian Pac J Cancer Prev, 15, 8529-38.

Marchetti C, Imperiale L, Gasparri ML, et al (2012). Olaparib, PARP1 inhibitor in ovarian cancer. Expert Opin Investig Drugs, 21, 1575-84.

Mougiakakos D, Choudhury A, Lladser A, et al (2010). Regulatory T cells in cancer. Adv Cancer Res, 107, 57-117.

Napoletano C, Bellati F, Landi R, et al (2010). Ovarian cancer cytoreduction induces changes in $\mathrm{T}$ cell population subsets reducing immunosuppression. J Cell Mol Med, 14, 2748-59.

Nielsen JS, Sahota RA, Milne K, et al (2012). CD20+ tumorinfiltrating lymphocytes have an atypical CD27- memory phenotype and together with CD8+ T cells promote favorable prognosis in ovarian cancer. Clin Cancer Res, 18, 3281-92.

Papadia A, Morotti M. (2013). Diaphragmatic surgery during cytoreduction for primary or recurrent epithelial ovarian cancer, a review of the literature. Arch Gynecol Obstet, 287, 733-41.

Paroli M, Bellati F, Videtta M, et al (2014). Discovery of chemotherapy-associated ovarian cancer antigens by interrogating memory $\mathrm{T}$ cells. Int J Cancer, 134, 1823-34.

Paulson KG, Carter JJ, Johnson LG, et al (2010).Antibodies to merkel cell polyomavirus $\mathrm{T}$ antigen oncoproteins reflect tumor burden in merkel cell carcinoma patients. Cancer Res, 70, 8388-97.

Raspollini MR, Castiglione F, Rossi Degl'innocenti D, et al (2005). Tumour-infiltrating gamma/delta T-lymphocytes are correlated with a brief disease-free interval in advanced ovarian serous carcinoma. Ann Oncol, 16, 590-6.

Salama M, Ormonde D, Quach T, et al (2010). Outcomes of endoscopic resection of large colorectal neoplasms, an Australian experience. J Gastroenterol Hepatol, 25, 84-9.

Santegoets SJ, Turksma AW, Suhoski MM, et al (2013). IL-21 promotes the expansion of $\mathrm{CD} 27+\mathrm{CD} 28+$ tumor infiltrating lymphocytes with high cytotoxic potential and low collateral expansion of regulatory T cells. J Transl Med, 11, 37.

Sato E, Olson SH, Ahn J, et al (2005). Intraepithelial CD8+ tumor-infiltrating lymphocytes and a high $\mathrm{CD} 8+/$ regulatory T cell ratio are associated with favorable prognosis in ovarian cancer. Proc Natl Acad Sci U S A, 102, 18538-43.

Schreiber RD, Old LJ, Smyth MJ et al (2011). Cancer immunoediting, integrating immunity's roles in cancer suppression and promotion. Science, 331, 1565-70.

Tomsová M, Melichar B, Sedláková I, et al (2008). Prognostic significance of CD3+ tumor-infiltrating lymphocytes in ovarian carcinoma. Gynecol Oncol, 108, 415-20.

Webb JR, Milne K, Watson P, et al (2014). Tumor-infiltrating lymphocytes expressing the tissue resident memory marker CD103 are associated with increased survival in high-grade serous ovarian cancer. Clin Cancer Res, 20, 434-44.

Yigit R, Massuger LF, Figdor CG, et al (2010). Ovarian cancer creates a suppressive microenvironment to escape immune elimination. Gynecol Oncol, 117, 366-72.

Zhang L, Conejo-Garcia JR, Katsaros D, et al (2003). Intratumoral T cells, recurrence, and survival in epithelial ovarian cancer. $N$ Engl J Med, 348, 203-13.

Zitvogel L, Kepp O, Kroemer G. (2011) Immune parameters affecting the efficacy of chemotherapeutic regimens. Nat Rev Clin Oncol, 8, 151-60. 